\title{
Polyurethane: A Versatile Scaffold for Biomedical Applications
}

\author{
Halima Khatoon and Sharif Ahmad* \\ Department of Chemistry, Jamia Millia Islamia (A Central University), India
}

*Corresponding author: Sharif Ahmad, Materials Research Laboratory, Department of Chemistry, Jamia Millia Islamia (A Central University) New, Delhi-110025, India

Submission: July 02, 2018; Published: July 19, 2018

\section{Introduction}

Polyurethanes (PUs) are one of the most versatile and explored polymeric materials in which the urethane groups are the major repeating unit and can be synthesized by reacting di or polyisocyanates (hard segments) with di or polyols (soft segments) via catalyzed polymerization process [1]. A broad range of PU with variety of physical and mechanical properties can be tuned just by changing the ratio of soft and hard segments [2]. Thus, it consists of two phase structure in which the hard segments are embedded into the soft segments. Using different ratios of hard/soft segments, it can be fabricated according to their need of applications in rubber, fibers, films, paints, coatings, elastomers, foams, gels etc. [3] These many forms of PUs available to date are simple improvements of the invention of Dr. Otto Bayer and his coworkers in the 1930s [4]. The continuous improvements in the polyurethane made them a suitable and promising material for the incorporation in widespread applications. For the decades, it has been used in the field of biomedical due to their well-known properties such as good durability, high tensile strength, fatigue resistance, excellent biodegradability and biocompatibility [5]. Among the polymers like, silicone, polyvinyl chloride (PVC), polyethylene and poly tetra fluoro ethylene (PTFE), PUs are widely used in medical application due to their superior bio and hemo compatibility. On account of this very characteristic property, they have extensively been used in catheters, heart valves, vascular grafts, prostheses and blood coagulating devices [6].

It has also been noticed that PUs are best suitable candidates for the scaffolds in tissue engineering, hydogels shape memory devices, nontoxic implants, various cardiovascular repair, wound healing and bone regenerations [7]. On account of this, the present review summarizes the chemistry of polyurethane, their historical background and considering factors for biomedical application. Moreover, their biomedical application in the field of tissue engineering and drug delivery has also been discussed.

\section{Historical Background}

The biomedical applications of PUs were first started in the late 1960s. In 1967, first generation polyurethane available industrially and commercially was used by Mirkovitch and groups as implantable biomaterials [8]. After that Sharp and coworkers have studied the thrombus resistance properties of polyester polyurethane for intravascular replacement [9]. A copolymer of polyurethane and polydimethyl siloxane was synthesized and used as blood compatible and heart assist balloon pumps was used by Nyilas and groups [10].

\section{Considering Factors for Biomedical Applications}

Biomedical application of any material is strictly related to their biocompatibility. Generally, when any foreign material comes in contact with the body tissues, a specific reaction occurs, which may give a negative response as a life threatening consequences [11]. Thus, in order to overcome these negative responses, PUs should have excellent biocompatibility, biodegradability and good mechanical properties. These properties can be tailored by the selection of monomer and the polymerization conditions. Although, PUs has all the properties, which are required for their biomedical applications, it can be engineered differently by using different soft/ hard segment ratios at the time of their synthesis [12]. For example, biodegradable PUs can easily make by incorporation of biodegradable segments into either the soft or hard segments of the polymer backbones. Hence, the selection of this ratio and polymerization condition is the main factor that must be consider for the application of PUs in biomedical. In the study reported by Sharifpoor et al. [13], a degradable polar ionic polyurethane was synthesized using lysine-based polycarbonate divinyl oligomer (DVO). Here the DVO was selected to optimize the physical properties and good biocompatibility. These properties suggest their potential applications for long term vascular tissue engineering.

\section{Biomedical Applications of Polyurethanes}

Owing to the excellent mechanical, biocompatible, biodegradable, high flexural endurance and fatigue resistance properties, PUs have become a material of choice for the development in biomedical applications. Here, we are discussing some of the important biomedical applications of PUs. 


\section{Tissue Engineering}

For the decades biodegradable PUs has been used for tissue engineering application due to their tunable mechanical properties, fatigue resistance and biocompatibility. It has been used in several tissue engineering such as fibro cartilage repair, bone regeneration, cardiac patches etc. The design of PUs as a scaffolds for tissue engineering requires with the development of bromidic properties that mimic the natural extracellular matrix and suitable to derive cell response. For this instance, many works have been carried out to design a PUs scaffolds for tissue engineering applications. Kishan et al. [14] have designed a tri block poly (ether ester) urethane using "plug and play" approach and studied their cycto compatibility and hydrolytic degradation to establish their application in tissue engineering. It was observed that the "plug and play" approach, used for the synthesis of biodegradable PU, gives a platform for highly tunable material aimed at tissue engineering application.

\section{Bone Regeneration}

As the bone regeneration involves a process like migration, proliferation, differentiation of osteo progenitors, and the formation of extracellular matrix, PU scaffolds have explored as potential candidate for this application. Recently, Wang et al. have synthesized biodegradable waterborne PU shape memory elastomer for bone tissue engineering [15]. First, they have prepared PU 3D printing ink in which polyethylene glycol (PEO) was used to adjust the viscosity. PU-gelatin 3D printing was also prepared for comparison, which showed good cell viability while the PU-PEO exhibited excellent shape memory properties. Thus, the PU scaffold with excellent shape memory property and osteogenic effect can be used as designer bone alternatives.

\section{Catheters}

PU Catheters are widely used in medical devices for the passage of fluids in the body. In general, they are in tube forms which are implanted into the body passage, canals and cavities. PUs, for their intrinsic flexibility, is widely used in intravenous and central vascular catheters. In the year 1980s, first PU catheter "Vialon ${ }^{\mathrm{TM} \text { " was }}$ developed. Later on, Smith and co-workers have used PU catheters for the absorption of drugs [16]

\section{Wound Dressing}

Another most important application of PUs is wound dressing. It is nontoxic, non-allergenic, and non-sensitizing, providing thermal insulation and mechanical protection and eventually heals the wounds without leaving any foreign particles in the wound. The healing process is generally carried out by upholding a wet environment at the wound interface and remove excess exudates to the surface of the dressing [17]. Gharibi et al. [18] have designed a membrane based on aniline tetramer embedded polyurethane-siloxane for wound dressing applications. The prepared membrane exhibited favorable properties for wound dressing such as water absorption, water vapor transmission rate and moist environment.

\section{Contact Lenses}

From the past few years, hydrophilic PU hydrogels are mainly used for their applications in contact lenses due to their good water absorption, high transparency and mechanical properties. The use of PU hydrogels were first disclosed by Blair and Hudgins [19]. The work reported by Gould and Johnson got patented in the year 1980 [20]. They have developed polyurethane diacrylate for their use in contact lenses. Their invention showed good water absorption, permeability to ions and gases and was stable in boiling water.

\section{Drug Delivery}

The unique chemistry and flexible processing of PUs made them a potential candidate as drug delivery carriers. For drug delivery application, PUs was used in the form of matrix, coatings and capsules in which anti-tumor drugs, proteins and antibiotics are introduced or adsorbed. However, it has been noticed that PUs are less compatibility with the blood and found unsuitable for in vivo drug delivery application [12]. To make them suitable and compatible with the blood, new generation PUs are now being developed with various modifications [21]. From the last few years, a great deal of research effort has been focused to modify the surface of PUs to improve their hemo compatibility. Yu et al. have synthesized a series of novel $\mathrm{pH}$ and reduction sensitive PU triblock copolymer for intracellular drug delivery [22]. It was observed that the in vitro drug release was significantly accelerated by reducing the $\mathrm{pH}$ or by addition of an intracellular reducing agent. Recently, Liu et al. have prepared a cross linked PU micelles with red ox sensitive and $\mathrm{pH}$ responsive surface charge switchable properties [21]. They have investigated that this novel PU micelles act as a drug delivery carriers due to their unique responsiveness to dual biological stimuli. In addition to aforementioned applications, biodegradable and biocompatible PUs have widened their application to the biomedical devices as well, which are listed in Table 1.

Table 1: Applications of Polyurethanes in various biomedical devices.

\begin{tabular}{|c|c|}
\hline Total Artificial Hearts & Fallopian Tubes \\
\hline Heart valves & Catheters and cannulas sutures \\
\hline Vascular prostheses & Ligaments \\
\hline Pericardial patches & Wound dressings \\
\hline Artificial heart or blood pumps & Shock absorbing elements \\
\hline Vascular Intra-aortic balloons & Pacemakers \\
\hline
\end{tabular}

\section{Conclusion and Future Perspectives}

This review summarizes history, design and properties suitable for biomedical applications of PUs. Moreover, the biomedical application in tissue engineering and drug delivery has also been explored. It was noted that biodegradable and biocompatible polyurethane has emerged as bio stable inert material and have used for broad range of biomedical applications. However, a new generation biocompatible and biodegradable polyurethane nano 
carrier is desired to develop to meet the therapeutic challenges and biological complexity. Furthermore, as the polymer structure plays an important role and determines the pharmaceutical properties, the optimization of synthesis chemistry and technology is needed to develop well-defined and well controlled polyurethane structures. As the applications studied in this review, it has been noticed that the drug delivery and in vivo properties of polyurethane has paid less attention. Thus there is a clear need to develop more animal studies and preclinical trials to accelerate the advancement of polyurethane and to gain the maximum possible benefits of biocompatible polyurethane in the upcoming future not only in the in the laboratory scale but also in the commercial level. Thus, we anticipate that the polyurethane can act as outstanding and game changing biomaterials that have the capability to expand and revolutionize its biomedical applications and have a positive impact in future.

\section{References}

1. Akindoyo JO, Beg MDH, Ghazali S, Islam MR, Jeyaratnam N, et al (2016) Polyurethane types, synthesis and applications-A review. RSC Adv 6(115): 114453-114482.

2. Zlatanic A, Lava C, Zhang W, Petrović ZS (2004) Effect of structure on properties of polyols and polyurethanes based on different vegetable oils. J Polym Sci Part B Polym Phys 42(5): 809-819.

3. Sharmin E, Zafar F (2012) Polyurethane: An introduction. Open access peer-reviewed chapter, UK, pp. 3-16.

4. Seymour R (1989) Pioneers in polymer science. Chemists and Chemistry, Germany, pp. 213-219.

5. Xu C, Yepez G, Wei Z, Liu F, Bugarin A (2016) Synthesis and characterization of conductive, biodegradable, elastomeric polyurethanes for biomedical applications. J Biomed Mater Res Part A 104(9): 2305-2314.

6. John WSP, Boretos W(1967) Segmented polyurethane: A new elastomer for biomedical applications. Science 158(3807): 1481-1482.
7. Erkesh LBR, Batyrbekov S, Moshkevich AS, Zhubanov BA (1990) Br Polym J 23: 213-276.

8. Mirkovitch KW, Akutsu T (1962) Trans Am Soc Artif Intern Organs 8: 79-84.

9. Wong EWC (1981) In urethane chemistry and application.

10. Nyilas E (1970) In $23^{\text {rd }}$ Conference on Engineering in Medicine and Biology. p. 147.

11. Habash M1 RG (1999) J Clin Pharmacol 39: 887-898.

12. Tan D, Li Z, Yao X, Xiang C,Tan H et al (2014) J Mater Chem B 2: 13441353.

13. Sharifpoor S, Labow RS, Santerre JP (2009) Biomacromolecules 10: 2729-2739.

14. Kishan AP, Wilems T, Mohiuddin S, Cosgriff HEM (2017) ACS Biomater Sci Eng 3: 3493-3502.

15. Wang YJ, Jeng US, Hsu SH (2018) ACS Biomater Sci Eng 4: 1397-1406.

16. Smith JC, Davies MC, Melia CD, Denyer SP, Derrick MR (1996) Biomaterials 17(15): 1469-1472.

17. Guo C, Zhou L , Lv J (2013) Polym Polym Compos 21: 449-456.

18. Gharibi R, Yeganeh H, Rezapour LA, Hassan ZM (2015 ACS Appl Mater Interfaces 7: 24296-24311.

19. Kurt CF, Daniel K (1996) Advances in Urethane: Science \& Technology. CRC Press, USA. Vol. 8.

20. Gould FE, Johnston CW (1980) Polyurethane diacrylate compositions useful for contact lenses and the like.

21. Liu C, Guan Y, Su Y, Zhao L, Meng F, et al. (2017) RSC Adv 7: 11021-11029.

22. Yu S, He C, Ding J, Cheng Y, Song W, et al. (2013) Soft Matter 9: 26372645.

Creative Commons Attribution 4.0

International License

For possible submissions Click Here Submit Article
SBB

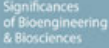

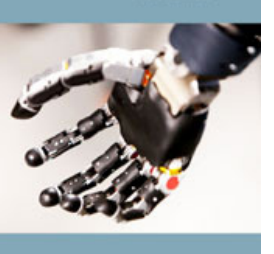

Significances of Bioengineering \& Biosciences

\section{Benefits of Publishing with us}

- High-level peer review and editorial services

- Freely accessible online immediately upon publication

- Authors retain the copyright to their work

- Licensing it under a Creative Commons license

- Visibility through different online platforms 